\title{
Emodin-loaded polymer-lipid hybrid nanoparticles enhance the sensitivity of breast cancer to Doxorubicin by inhibiting epithelial mesenchymal transition
}

\author{
Tengteng Zou \\ Jinan University \\ Meng Lan \\ Jinan University \\ Lihong Li \\ Jinan University \\ Tiange Cai \\ Liaoning University \\ Yu Cai ( $\nabla$ caiyu8@sohu.com) \\ Jinan University
}

\section{Research}

Keywords: Emodin, polymeric lipid nanoparticles, breast cancer chemoresistance, Doxorubicin, Epithelial mesenchymal transition

Posted Date: May 13th, 2021

DOl: https://doi.org/10.21203/rs.3.rs-451946/v1

License: (c) (1) This work is licensed under a Creative Commons Attribution 4.0 International License.

Read Full License

Version of Record: A version of this preprint was published at Cancer Nanotechnology on August 11th, 2021. See the published version at https://doi.org/10.1186/s12645-021-00093-9. 


\section{Abstract \\ Background}

The role of epithelial mesenchymal transition (EMT) involved in breast cancer metastasis and chemoresistance has been increasingly recognized. However, it's necessary to search for more effective strategies to inhibit EMT thereby increase the sensitivity of breast cancer cells to chemotherapy drugs. Emodin has a potential in overcoming tumor drug resistance and restraining the development of EMT, but the poor internalization into breast cancer cells limited the application.

\section{Results}

MCF-7/ADR cells have more EMT characteristics than MCF-7 cell. EMT in MCF-7/ADR cells promotes the development of drug resistance via apoptosis resistance and facilitating the expression of P-gp. The anticancer effect of DOX enhanced by the decreasing of drug resistance protein P-gp and apoptosis related proteins after EMT inhibited in MCF-7/ADR cells. E-PLNs increase the cellular uptake of EMO and restore DOX sensitivity in MCF-7/ADR cells by inhibiting EMT.

\section{Conclusion}

E-PLNs inhibit EMT to enhance the sensitivity of breast cancer to DOX. The combination of E-PLNs and DOX can improve the efficacy of DOX in the treatment of breast cancer, which provides a new method to prevent or delay clinical drug resistance.

\section{Background}

Despite significant advances in cancer diagnosis and treatment, breast cancer to be the number one cancer in the world by 2020 , more than $90 \%$ of the death causes of breast cancer patients are related to chemotherapy resistance (Siegel et al. 2021, Sung et al. 2021). The role of epithelial mesenchymal transition (EMT) involved in chemotherapy resistance has been increasingly recognized. Asiedu MK and his colleagues found that EMT induced by TGF- $\beta$ pathway in breast cancer cells decreased the sensitivity to Doxorubicin (DOX) and paclitaxel (Asiedu et al. 2014). Reversal tumor drug resistance through EMT inhibition is a new strategy and search for a safe and effective EMT inhibitor is necessary.

Emodin (EMO), the active component of Rheum rhabarbarum, is proved to have antitumor activity in breast cancer (Sun et al. 2015). In addition, EMO has a potential in overcoming tumor drug resistance and restraining the development of EMT (Liu et al. 2020, Fu et al. 2012, Lu et al. 2016, Gu et al. 2019). It can effectively restore the effect of DOX, vincristine and other anticancer drugs by downregulating the expression of P-gp in MCF-7/ADR cells. It was found that the combination of DOX and EMO effectively inhibit the expression of snail, twist and slug, thereby inhibiting cell proliferation and promote cell 
apoptosis. This combination results the suppression of cell migration and invasion. However, the poor internalization into the breast cancer cells limited the application of EMO. Hence, improving EMO internalization in breast cancer cells tend to be a reliable way to enhance the anti-cancer effect of DOX. Furthermore, the drug reversal effect of EMO whether rely on the repression of EMT still needed to be elucidated.

Nanoparticles loaded with anticancer drugs become an ideal formulation for reversing chemoresistance (Li et al. 2016, Markman et al. 2013). The tumor targeting effect of nanoparticles is basely depended on the enhanced permeability and retention (EPR) in tumor microenvironment (TME), thus drug accumulation increased in TME (Kalyane et al. 2019). Polymeric lipid nanoparticles (PLNs), designed to combine the advantages of both liposomes and polymeric nanoparticles, can meet the structural and property differences of various anticancer drugs, as well as the combination therapy strategy, making them a promising drug carrier (Jain et al. 2020, Mukerjee, Ranjan and Vishwanatha 2012).

Targeting EMT is becoming one of the new strategies for drug resistance reversal in various tumors. In this study, the relationship of EMT and drug resistance were discussed. Here, we provided evidence for EPLNs to improve the therapeutic effect of DOX on breast cancer. Further, the evidence concerning drug resistance reversal mechanism of E-PLNs in suppressing EMT is provided.

\section{Results}

\section{EMT is associated with DOX resistance in breast cancer cells}

We compared the effect of DOX on the viability of MCF-7 cells and MCF-7/ADR cells. As Fig. 1A, the toxicity of DOX on MCF-7/ADR cells than MCF-7 cells were less, IC50 was $5.35 \mu \mathrm{M}$, while the IC50 of MCF-7/ADR cells were 124.1 $\mu \mathrm{M}$. MCF-7/ADR cells were resistant to DOX with a resistance index of 23.2. EMT is characterized by loss of E-cadherin expression and upregulated Vimentin and N-cadherin (Fig. 1B and C). DOX induced drug resistant cells have EMT characteristics, and the ability of invasion significantly enhanced (Fig. 1D and E).

\section{EMT inhibiting cell proliferation and invasion}

TGF-beta signaling has been shown to play an important role in EMT. Galunisertib, a selective TGF- $\beta$ receptor type I (TGF- $\beta$ RI) kinase inhibitor to block TGF- $\beta$ signaling reverse EMT, is applicated in this part to investigate the relationship of EMT, cell proliferation and invasion. It can be seen from Fig $2 A$ that different concentrations of Galunisertib can inhibit the proliferation of MCF-7/ADR cells, and the IC50 value calculated is $248.2 \mu \mathrm{M}$. When the concentration of Galunisertib was $30 \mu \mathrm{M}$, the cell viability was more than $90 \%$. Therefore, the concentration of $30 \mu \mathrm{M}$ Galunisertib is selected to application for the subsequent experiment to exclude the interference of Galunisertib cytotoxicity. Galunisertib reversed EMT marker protein expression in the selected concentration (Fig. 2B and C). The therapeutic effect of DOX was improved under the incubation of $10 \mu \mathrm{M}$ DOX and $30 \mu \mathrm{M}$ Galunisertib (Fig. 2D) MCF-7/ADR cells have higher invasive ability than the DOX sensitive MCF-7 cells, but invasive ability of MCF-7/ADR cells 
treated with DOX did not change. Compared with DOX $(10 \mu \mathrm{M})$ group, Galunisertib $(30 \mu \mathrm{M})$ group cells was decreased and the invasion activity was inhibited. When Galunisertib and DOX were used together, the inhibition effect was stronger (Fig. 2E and F). These results suggest that the inhibition of EMT may weaken the drug resistance of MCF-7/ADR cells.

\section{Blocking EMT restrain the expression of P-gp and induced apoptosis}

Apoptosis is an important manifestation of anti-tumor effect of chemotherapy drugs. We can see from Fig $3 A$ and $B$ that the apoptosis rate of DOX $(10 \mu \mathrm{M})$ group is only $13.81 \%$, Galunisertib $(30 \mu \mathrm{M})$ group (the apoptosis rate is $7.85 \%$ ) has little effect on apoptosis. When DOX was combined with Galunisertib, the number of apoptotic cells increased, and the apoptotic rate was $23.88 \%$. It can be seen from Fig. $3 \mathrm{C}$ and $D$ that compared with DOX group, Bax/Bcl-2 ratio increased in DOX+Galunisertib (30 $\mu \mathrm{M})$ group. These results suggest that inhibition of EMT can enhance DOX induced apoptosis in MCF-7/ADR cells.

EMT was closely related to the process of drug resistance, one of which was upregulation of drug resistance related transporters. As can be seen in Fig. 3E and F, the difference of expression level of P-gp protein in Control group and DOX group was not significant. However, when MCF-7/ADR cells were treated with Galunisertib, the expression levels of P-gp protein were down-regulated. More importantly, the expression of P-gp in DOX+Galunisertib group was significantly suppressed than DOX group, resulting in decreasing of DOX efflux. Therefore, blocking EMT down-regulate the expression level of P-gp, thereby restraining the DOX efflux in MCF-7/ADR cells.

\section{E-PLNs increase the cellular uptake and cytotoxity of EMO}

E-PLNs prepared by nano precipitation method are light yellow liquid (Fig. 4A). In order to preserve the samples for a long time, we keep E-PLNs samples through freeze-drying (Christ ALPHA 1-4 LD plus). The particle size of E-PLNs was $122.7 \pm 1.03 \mathrm{~nm}$ (Fig. 4C), zeta potential was $-28.5 \pm 1.55 \mathrm{mV}$ (Fig. 4D) and PDI was 0.118 . The results show that the nanoparticles have uniform size and good stability.

As E-PLNs have no fluorescence, the C6-PLNs, characterized by green fluorescence, is formulated to analyze the cellular uptake of PLNs carriers. After the coumarin-6-labeled nanoparticles were internalized, the green fluorescence mainly distributed in the cytoplasm (Fig. 5A), indicating that PLNs carriers promote the cellular uptake of the loaded drugs. Furthermore, flow cytometry quantitatively analyzes cellular uptake, and the uptake intensity was expressed as the average fluorescence intensity of cells. The average fluorescence intensity of cells was 500 , indicating that the cells had a high uptake of nanoparticles (Fig 5B and C). The cytotoxicity of EMO group (Fig.5D) and E-PLNs (Fig.5E) group on MCF7/ADR cells gradually increased with the increase of EMO concentration. The IC50 of EMO and E-PLNs were $150.5 \mu \mathrm{M}$ and $138.7 \mu \mathrm{M}$, respectively, which indicated that E-PLNs could enhance the antitumor activity of EMO. In order to further explore whether EMO or E-PLNs can enhance the toxicity of DOX, a suitable concentration was selected and combined with DOX to detect the effect on cell viability (Fig.5F). We selected $30 \mu \mathrm{M}$ EMO and E-PLNs for the subsequent combined administration experiment. It can be 
seen from Fig. that $30 \mu \mathrm{M}$ E-PLNs can enhance the cytotoxicity of different concentrations of DOX. Finally, $10 \mu \mathrm{M}$ DOX was selected to explore how E-PLNs improve the therapeutic effect of DOX.

\section{E-PLNs enhance the effect of DOX in apoptosis induction}

MCF-7/ADR cells were treated with DOX, DOX+EMO, DOX+E-PLNs to investigate whether E-PLNs induced cell apoptosis. The morphological changes of apoptotic cells are shown in Fig $6 \mathrm{~A}$. We observed that the untreated cells were round or oval blue nuclei, while the cells in the treatment group were fragmented and apoptotic cells were formed, and the number of apoptotic bodies was significantly reduced in the E-PLNs group. It can be seen from Fig. 6B and C that compared with DOX $(10 \mu \mathrm{M})$ group $(15.52 \%)$, the apoptosis rates of DOX+EMO $(30 \mu \mathrm{M})$ and DOX+E-PLNs $(30 \mu \mathrm{M})$ groups were $18.49 \%$ and $24.48 \%$, respectively, and the apoptosis rate was significantly increased. Western blot results also showed that the ratio of $\mathrm{Bax} / \mathrm{Bcl}-$

2 was increased and the expression of Fas was up-regulated in DOX+EMO $(30 \mu \mathrm{M})$ and DOX+E-PLNs (30 $\mu \mathrm{M})$ groups, which promoted cell apoptosis. At the same time, the expression level of Bcl-2 was down regulated. DOX+E-PLNs group showed a more significant effect on the expression of apoptotic protein (Fig. 6D and E). Flow cytometry combined with Western blot showed that E-PLNs may enhance the apoptosis inducing effect of DOX on MCF-7/ADR cells through affecting apoptosis related pathways.

\section{E-PLNs enhance DOX accumulation and restrain the expression of P-gp}

The accumulation of DOX in MCF-7/ADR cells treated by different administration groups (Control, DOX, DOX+EMO, DOX+E-PLNs) was analyzed quantitatively by flow cytometry (Fig. 7A). From Fig. 7B, it can be seen that the peak map of DOX+EMO $(30 \mu \mathrm{M})$ and DOX+E-PLNs $(30 \mu \mathrm{M})$ groups shifts to the direction of high fluorescence intensity compared with DOX $(10 \mu \mathrm{M})$ group. The average fluorescence intensity of DOX+E-PLNs is higher than that of DOX group囚indicated that E-PLNs promote the uptake of DOX and increase the DOX accumulation in MCF-7/ADR cells. In addition, the expression of P-gp significantly decreased in DOX+EMO and DOX+E-PLNs groups compared with control and DOX groups (Fig 7C and D), indicated that E-PLNs could effectively inhibit the expression of P-gp protein thus enhancing the effect of DOX.

\section{E-PLNs inhibit the expression of EMT marker protein and cell invasion}

The expression of E-cadherin decreased, while the expression of $\mathrm{N}$-cadherin and Vimentin increased in MCF-7/ADR cells, which indicated the occurrence of EMT. It can be seen from Fig. $8 \mathrm{~A}$ and $\mathrm{B}$ that there is no significant difference in the protein levels of E-cadherin and Vimentin between the Control group and the DOX group, but the protein level of N-cadherin increases significantly, which indicates that DOX administration to MCF-7/ADR cells may further promote EMT. In DOX+E-PLNs $(30 \mu \mathrm{M})$ group, the expression of E-cadherin protein was increased, and the expression of vimentin and $\mathrm{N}$-cadherin protein was up-regulated. These results indicate that E-PLNs can effectively inhibit EMT, and the enhancement of DOX sensitivity of MCF-7/ADR cells by E-PLNs is related to the inhibition of EMT. 
Cell invasion is an important manifestation after EMT induction. In Fig. $8 \mathrm{C}$ and D, there was no significant difference in the number of cell invasion between the Control group and DOX group. Compared with DOX group, the number of invasive cells in DOX+EMO $(30 \mu \mathrm{M})$ group and DOX+E-PLNs $(30 \mu \mathrm{M})$ group decreased, and DOX+E-PLNs group was more significant. These results shown that the combination of EPLNs and DOX can inhibit the occurrence of EMT and the tendency to invasion in MCF-7/ADR cells.

\section{Discussion}

Chemotherapy is the main treatment for early stage of breast cancer, but the sensitivity of tumor cells to chemotherapeutic drugs decreases with long-term chemotherapy. Increasing evidence shown that EMT is involved in the development of drug resistance in breast cancer (Jiang et al. 2017). After EMT was induced by snail eukaryotic expression vector, the expression of BCRP were significantly increased and the drug resistance of MCF-7 cells was increased to 9.93 times (Du and Shim 2016). In this article, we find that appears MCF-7/ADR cells have more significant characteristics of EMT than DOX sensitive MCF7 cells, manifested as the down-regulated of E-cadherin protein and the up-regulated of $\mathrm{N}$-cadherin protein and Vimentin. In fact, $\mathrm{Li}$ and his colleagues also confirmed that DOX could induce EMT and apoptosis in MCF-7 cells ( $\mathrm{Li}$ et al. 2020b). The invasion and metastasis are significantly enhanced in tumor cells with EMT characteristics. Indeed, the invasion test showed that MCF-7/ADR cells had more invasive cells than MCF-7 cells, which proved that MCF-7/ADR cells had stronger invasion ability.

Transforming growth factor-beta (TGF- $\beta$ ) signaling plays a key role in epithelial-mesenchymal transition (EMT) of tumors (Xu, Lamouille and Derynck 2009). Galunisertib is a selective TGF- $\beta$ receptor type I (TGF$\beta R I)$ kinase inhibitor which blocking TGF- $\beta$ signaling reverse EMT (Xu et al. , Rodón et al. 2015). The direct toxicity of DOX to cells was enhanced by Galunisertib, which decreased cell viability and increase cell invasion. Moreover, the apoptosis induction in MCF-7/ADR cells of DOX restored after EMT suppression. In addition, previous studies have shown that both P-gp and ATP binding cascade transporters are regulated by EMT. In this study, as the number of cells with EMT characteristics were decreased, and the expression of P-gp protein was restrained. Therefore, it is worth to confirm that EMT participates in the drug resistance of cancer cells by regulating the processes of apoptosis and drug resistance proteins expression.

EMO has been found to inhibit to increase sensitivity to DOX and PTX in breast cancer ( $L i$ et al. 2020b, Gu et al. 2020), and inhibits EMT in triple negative breast cancer (Song et al. 2018). It's reported that solid lipid nanoparticles of EMO can significantly block the cell cycle and induce apoptosis of MCF-7 cells compared with free EMO (Wang et al. 2012), which indicates that lipid nanoparticles delivering EMO may be a promising method for cancer treatment. In this research, we prepared PLNs encapsulated EMO to enhance cellular uptake. As E-PLNs have no fluorescence, the C6-PLNs is formulated to analyze the cellular uptake of PLNs carriers. The result shown that C6-PLNs can be absorbed by MCF-7/ADR cells, and quantitative analysis of the average fluorescence intensity shown that PLNs structure improve the uptake of C6. Furthermore, the cellular uptake and anti-tumor effect of DOX improved with the combination of E-PLNs, indicated that EMO enhanced the therapeutic effect of DOX on tumor cells. The 
main mechanisms of the anti-cancer effect in DOX is apoptosis induction, and the apoptosis rate increased to $24.48 \%$ combined with E-PLNs. The apoptotic pathway was activated by the increase of $\mathrm{Bax} / \mathrm{Bcl}-2$ ratio. The expression of P-gp is restrained, resulting in the decrease of DOX efflux. The upregulated expression of E-cadherin, and down-regulated N-cadherin and Vimentin in MCF-7/ADR cells incubated with E-PLNs indicate that E-PLNs inhibit EMT, therefore reverse DOX resistance in MCF-7/ADR cells.

In this study, we find that EMT is involved in drug resistance, which is related to the regulation of apoptosis and the expression of drug resistance transporters. EMT is one of the key factors to induce DOX resistance in breast cancer. We also found that the expression of E-cadherin in DOX+E-PLNs group was higher than that in DOX group, while the expression of Vimentin and N-cadherin was down regulated. In addition, when E-PLNs were combined with DOX, the number of invasive cells decreased significantly. These results suggested that E-PLNs enhancing the sensitivity of breast cancer to DOX depended on inhibiting EMT.

\section{Conclusion}

Drug resistance in tumor is a phenomenon which the continuous administration of chemotherapeutic drugs induces drug tolerance. EMT is one of the possible ways causing DOX resistance in breast cancer. Our data suggested that the DOX resistance in breast cancer is associated with EMT. E-PLNs enhance the sensitivity of DOX in breast cancer via the suppression of EMT. We confirmed that E-PLNs enhance the effect of DOX, which is closely related to the regulation of EMT thereby inhibiting of apoptosis resistance and P-gp expression. The combination of E-PLNs and DOX provides a new scheme for clinical treatment. It's potential that E-PLNs turn into an adjuvant therapy drugs of breast cancer in the feature. Further investigations in animal models and mechanism are warranted.

\section{Materials And Methods}

\section{Materials}

Emodin ( $>98 \%$ purity) were provided by institute for drug control (Guangzhou, China). Mannitol was purchased from Aladdin Biotech Co., Ltd. (Shanghai, China). Doxorubicin hydrochloride was obtained from Selleck Chemicals (TX, USA). Galunisertib (LY2157299) were obtained from Beyotime Biotechnology (Shanghai, China). P-glycoprotein primary antibodies and was purchased from Abcam (Cambridge, UK). Annexin V-APC dye was provided by KeyGEN BioTECH (Jiangsu, China). Propidium iodide (PI) staining buffer was obtained from BD Biosciences Pharmingen (Shanghai, China). E-cadherin, $\mathrm{N}$-cadherin and Vimentin primary antibodies were purchased from CST, Inc (Massachusetts, USA). Human breast MCF-7 cells and MCF-7/ADR human breast cancer DOX resistant cells were purchased from KeyGen BioTECH (Jiangsu, China).

\section{Preparation of E-PLNs lyophilized powder}


We prepared E-PLNs by nano precipitation method (Tahir et al. 2019). Briefly, EMO and PLGA were dissolved in acetone. Another soybean phospholipid and DEPE-PEG 2000 were dissolved in 5\% (W/V) F68 aqueous solution. The organic phase was slowly and evenly added to Pluronic F-68 aqueous solution at $75^{\circ} \mathrm{C}$, and stirred (8000 rpm for $40 \mathrm{~min}$ ) until the acetone completely evaporated. The supernatant was collected by centrifugation (1000 rpm for $5 \mathrm{~min}$ ). The particle size and Zeta potential of E-PLNs were measured by Malvern Zetasizer Nano ZS90. The prepared E-PLNs were added with 10\% (W/V) freezedrying protective agent (Mannitol). After being frozen overnight at $-80^{\circ} \mathrm{C}$, E-PLNs were freeze-dried for 24 h and stored at $4{ }^{\circ} \mathrm{C}$.

\section{Cell culture}

MCF-7 cells were cultured in 10\% FBS of RPMI-1640 medium under defined circumstances. In order to maintain cell resistance to DOX, MCF-7/ADR cells were cultured in RPMI-1640 medium containing DOX $(250 \mathrm{ng} / \mathrm{mL})$ every third passage.

\section{Cellular uptake}

Coumarin-6 $(200 \mu \mathrm{g} / \mathrm{mL})$ was encapsulated in PLNs instead of EMO to prepare coumarin 6-PLNs (C-6 PLNs). MCF/ADR were incubated with C- 6 PLNs for $2 \mathrm{~h}$, fixed by $4 \%$ paraformaldehyde, and treated with Hochest staining solution for $5 \mathrm{~min}$. The uptake of C-6 PLNs in MCF/ADR was observed under laser confocal microscope and analyzed by Flow cytometry.

\section{MTT assay}

Cells were cultured in 96 well plate for $24 \mathrm{~h}$. Then the fresh culture medium containing free DOX, free EMO, E-PLNs, DOX+EMO, DOX+E-PLNs were added to the culture medium, and cultured for more than $48 \mathrm{~h}$ for cell test. After incubation with MTT $(5 \mathrm{mg} / \mathrm{mL}, 20 \mu \mathrm{L} /$ well $)$ for $4 \mathrm{~h}$, the supernatant was removed. The cells were exposed to DMSO solution $(150 \mu \mathrm{L} /$ well $)$ and incubated for $15 \mathrm{~min}$. Finally, the OD value was detected (Microplate Reader, $490 \mathrm{~nm}$ ) and cell viability was calculated.

\section{Cell invasion assay}

Cells were resuspended in RPMI-1640 medium without FBS, then seed in the upper chamber for $24 \mathrm{~h}$, and $500 \mu \mathrm{L}$ culture medium containing different drugs was added into the lower chamber. Cells were fixed with $4 \%$ paraformaldehyde for 10 min and stained with $200 \mu \mathrm{L}$ crystal violet staining solution for $15 \mathrm{~min}$. 5 fields of vision were randomly selected under a $100 \times$ microscope to observed the cells passing through the filter membrane. After taking photos, $500 \mu \mathrm{L} 33 \%$ acetic acid solution was added, shaking for $10 \mathrm{~min}$, the $O D$ value was measured to indirectly reflect the number of invasive cells.

\section{Apoptosis study}

MCF-7/ADR cells ( $3 \times 105$ cells/well, 6 well plate) were cultured, then treated with fresh medium containing different drugs for $48 \mathrm{~h}$. The cells were digested with trypsin without EDTA. After centrifugation again, 
cells were suspend with $500 \mu \mathrm{L}$ Binding Buffer. Then, Annexin-V/FITC and PI staining solution was added with $5 \mu \mathrm{L}$ each and mixed thoroughly. All experimental groups were sincubated in the dark for 15 min. Finally, the apoptosis was detected on the flow cytometer.

\section{Western blotting analysis}

Treated with different treatments for $48 \mathrm{~h}$, cells were lysed with lysate, and the protein was obtained by centrifugation (10000 rpm for $5 \mathrm{~min}$ ). BCA method was used to determine the protein concentration. After the expressed proteins ( $30 \mu \mathrm{g}$ each) were separated by SDS-PAGE (Bio RAD), and transferred to PVDF membrane. The unbound protein sites were blocked with $5 \%$ skim milk for $2 \mathrm{~h}$. The PVDF membrane was incubated with solution containing with different primary antibodies at $4{ }^{\circ} \mathrm{C}$ overnight. After washed with PBST for $1 \mathrm{~h}$, The PVDF membrane incubated with solution containing horseradish peroxidase bound secondary antibody for $2 \mathrm{~h}$. The PVDF membrane was evenly coated with ECL developer. According to the manual, the exposure was carried out with chemiluminescence imaging system and analyzed with Image J software.

\section{Statistical analysis}

The experiment was repeated three times, and the average value \pm SD value was calculated. GraphPad Prism software analyzes and draws pictures, and the ONE-WAY ANOVE analysis is used for significance. $* p<0.05, * \star p<0.01$, and $* \star * p<0.001$ are considered to be significantly different.

\section{Declarations}

\section{Ethics approval and consent to participate}

Not Applicable.

\section{Consent for publication}

All authors agree with the publication of this manuscript in this journal.

\section{Availability of data and materials}

The data will be available if needed.

\section{Competing interests}

The authors declare that there is no conflict of interest

\section{Funding}

This work was supported by the Natural Science Foundation of Guangdong (2021A1515011241).

\section{Authors' contributions}


Zou designed the experimental protocols, performed the experiments and analyzed the results. Lan, Li, Tiange Cai, and Yu Cai contributed to writing the manuscript. All authors reviewed the final draft of the manuscript. All authors read and approved the final manuscript.

\section{Acknowledgements}

Authors acknowledges the Natural Science Foundation of Guangdong for providing financial support to carry out the work under the funding 2021A1515011241.

\section{Abbreviations}

EMT: epithelial mesenchymal transition; EMO: Emodin; E-PLNs: Emodin-loaded polymer-lipid hybrid nanoparticles; DOX: Doxorubicin; TGF- $\beta$ : Transforming growth factor beta; TME: Tumor microenvironment; P-gp: P-glycoprotein; PDI: Polydispersity index; Bcl-2: B-cell lymphoma 2; Bax: Bcl-2associated X protein; GAPDH: Glyceraldehyde 3-phosphate dehydrogenase; PI: Propidium iodide; DAPI: 4',6-diamidino-2-phenylindole; PLGA: Poly (lactic-co-glycolic acid).

\section{References}

Siegel RL, Miller KD, Fuchs HE, et al. Cancer Statistics, 2021. CA Cancer J Clin. 2021 Jan;71(1):7-33.

Sung H, Ferlay J, Siegel RL, et al. Global cancer statistics 2020: GLOBOCAN estimates of incidence and mortality worldwide for 36 cancers in 185 countries. CA Cancer J Clin. 2021 Feb 4.

Asiedu, M. K., F. D. Beauchamp-Perez, J. N. Ingle, M. D. Behrens, D. C. Radisky \& K. L. Knutson (2014) AXL induces epithelial-to-mesenchymal transition and regulates the function of breast cancer stem cells. Oncogene, 33, 1316-24.

Sun, Y., X. Wang, Q. Zhou, Y. Lu, H. Zhang, Q. Chen, M. Zhao \& S. Su (2015) Inhibitory effect of emodin on migration, invasion and metastasis of human breast cancer MDA-MB-231 cells in vitro and in vivo. Oncol Rep, 33, 338-46.

Liu, Q., J. Hodge, J. Wang, Y. Wang, L. Wang, U. Singh, Y. Li, Y. Yao, D. Wang, W. Ai, P. Nagarkatti, H. Chen, P. Xu, E. A. Murphy \& D. Fan (2020) Emodin reduces Breast Cancer Lung Metastasis by suppressing Macrophage-induced Breast Cancer Cell Epithelial-mesenchymal transition and Cancer Stem Cell formation. Theranostics, 10, 8365-8381.

Lu, J., Y. Xu, X. Wei, Z. Zhao, J. Xue \& P. Liu (2016) Emodin Inhibits the Epithelial to Mesenchymal Transition of Epithelial Ovarian Cancer Cells via ILK/GSK-3ß/Slug Signaling Pathway. Biomed Res Int, $2016,6253280$.

Gu, J., C. F. Cui, L. Yang, L. Wang \& X. H. Jiang (2019) Emodin Inhibits Colon Cancer Cell Invasion and Migration by Suppressing Epithelial-Mesenchymal Transition via the Wnt/ $\beta$-Catenin Pathway. Oncol Res, 
Fu, J. M., J. Zhou, J. Shi, J. S. Xie, L. Huang, A. Y. Yip, W. T. Loo, L. W. Chow \& E. L. Ng (2012) Emodin affects ERCC1 expression in breast cancer cells. J Trans/ Med, 10 Suppl 1, S7.

Song, X., X. Zhou, Y. Qin, J. Yang, Y. Wang, Z. Sun, K. Yu, S. Zhang \& S. Liu (2018) Emodin inhibits epithelial-mesenchymal transition and metastasis of triple negative breast cancer via antagonism of CC-chemokine ligand 5 secreted from adipocytes. Int J Mol Med, 42, 579-588.

Li, W., H. Zhang, Y. G. Assaraf, K. Zhao, X. Xu, J. Xie, D. H. Yang \& Z. S. Chen (2016) Overcoming ABC transporter-mediated multidrug resistance: Molecular mechanisms and novel therapeutic drug strategies. Drug Resist Updat, 27, 14-29.

Markman, J. L., A. Rekechenetskiy, E. Holler \& J. Y. Ljubimova (2013) Nanomedicine therapeutic approaches to overcome cancer drug resistance. Adv Drug Deliv Rev, 65, 1866-79.

Kalyane, D., N. Raval, R. Maheshwari, V. Tambe, K. Kalia \& R. K. Tekade (2019) Employment of enhanced permeability and retention effect (EPR): Nanoparticle-based precision tools for targeting of therapeutic and diagnostic agent in cancer. Mater Sci Eng C Mater Biol Appl, 98, 1252-1276.

Jain, V., H. Kumar, H. V. Anod, P. Chand, N. V. Gupta, S. Dey \& S. S. Kesharwani (2020) A review of nanotechnology-based approaches for breast cancer and triple-negative breast cancer. $\mathrm{J}$ Control Release, $326,628-647$.

Mukerjee, A., A. P. Ranjan \& J. K. Vishwanatha (2012) Combinatorial nanoparticles for cancer diagnosis and therapy. Curr Med Chem, 19, 3714-21.

Jiang, Z. S., Y. Z. Sun, S. M. Wang \& J. S. Ruan (2017) Epithelial-mesenchymal transition: potential regulator of $A B C$ transporters in tumor progression. J Cancer, 8, 2319-2327.

Du, B. \& J. S. Shim (2016) Targeting Epithelial-Mesenchymal Transition (EMT) to Overcome Drug Resistance in Cancer. Molecules, 21.

Li, S., Y. Fan, A. Kumagai, E. Kawakita, M. Kitada, K. Kanasaki \& D. Koya (2020b) Deficiency in Dipeptidyl Peptidase-4 Promotes Chemoresistance through the CXCL12/CXCR4/mTOR/TGF $\beta$ Signaling Pathway in Breast Cancer Cells. Int J Mol Sci, 21.

Xu, J., S. Lamouille \& R. Derynck (2009) TGF-beta-induced epithelial to mesenchymal transition. Cell Res, $19,156-72$.

Rodón, J., M. Carducci, J. M. Sepulveda-Sánchez, A. Azaro, E. Calvo, J. Seoane, I. Braña, E. Sicart, I. Gueorguieva, A. Cleverly, N. S. Pillay, D. Desaiah, S. T. Estrem, L. Paz-Ares, M. Holdhoff, J. Blakeley, M. M. Lahn \& J. Baselga (2015) Pharmacokinetic, pharmacodynamic and biomarker evaluation of transforming 
growth factor- $\beta$ receptor I kinase inhibitor, galunisertib, in phase 1 study in patients with advanced cancer. Invest New Drugs, 33, 357-70.

Gu, M., W. Zheng, M. Zhang, X. Dong, Y. Zhao, S. Wang, H. Jiang \& X. Zheng (2020) LncRNA NONHSAT141924 promotes paclitaxel chemotherapy resistance through p-CREB/Bcl-2 apoptosis signaling pathway in breast cancer. $J$ Cancer, 11, 3645-3654.

Song, X., X. Zhou, Y. Qin, J. Yang, Y. Wang, Z. Sun, K. Yu, S. Zhang \& S. Liu (2018) Emodin inhibits epithelial-mesenchymal transition and metastasis of triple negative breast cancer via antagonism of CC-chemokine ligand 5 secreted from adipocytes. Int J Mol Med, 42, 579-588.

Wang, S., T. Chen, R. Chen, Y. Hu, M. Chen \& Y. Wang (2012) Emodin loaded solid lipid nanoparticles: preparation, characterization and antitumor activity studies. Int $J$ Pharm, 430, 238-46.

Tahir, N., A. Madni, A. Correia, M. Rehman, V. Balasubramanian, M. M. Khan \& H. A. Santos (2019) Lipidpolymer hybrid nanoparticles for controlled delivery of hydrophilic and lipophilic doxorubicin for breast cancer therapy. Int J Nanomedicine, 14, 4961-4974.

\section{Figures}


(A)

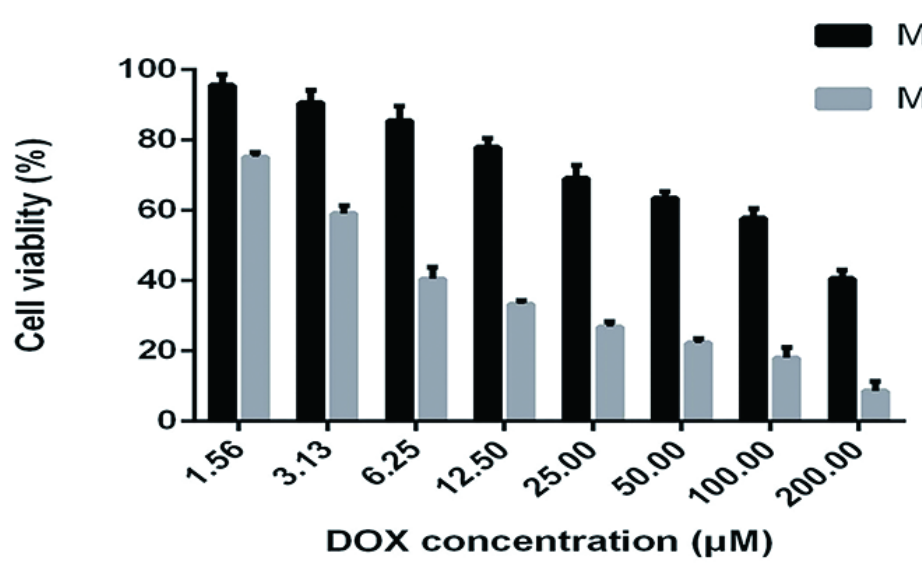

(B)

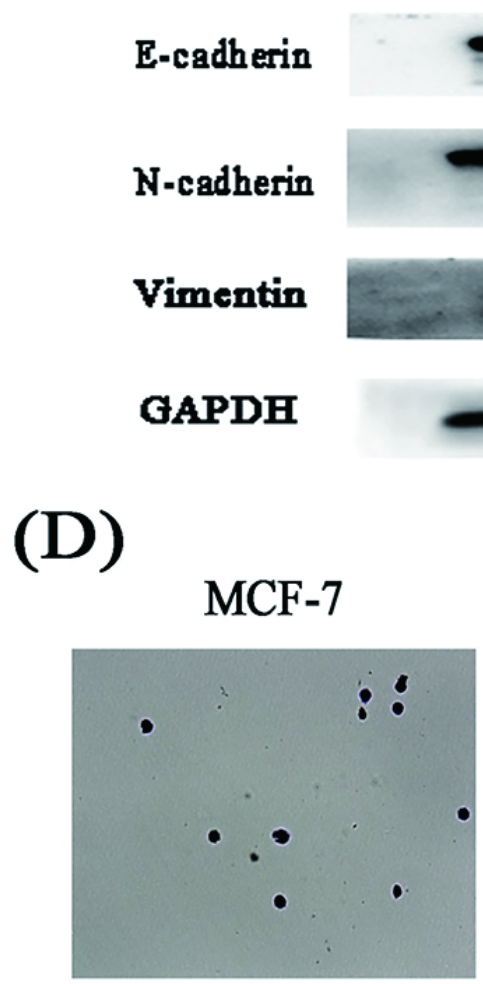

(C)

\section{MCF-7 MCF-7/ADR}
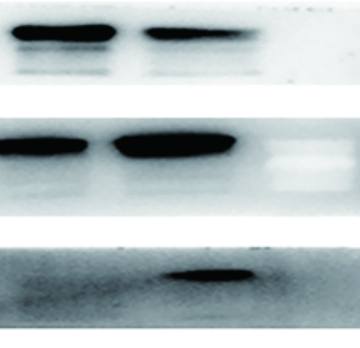

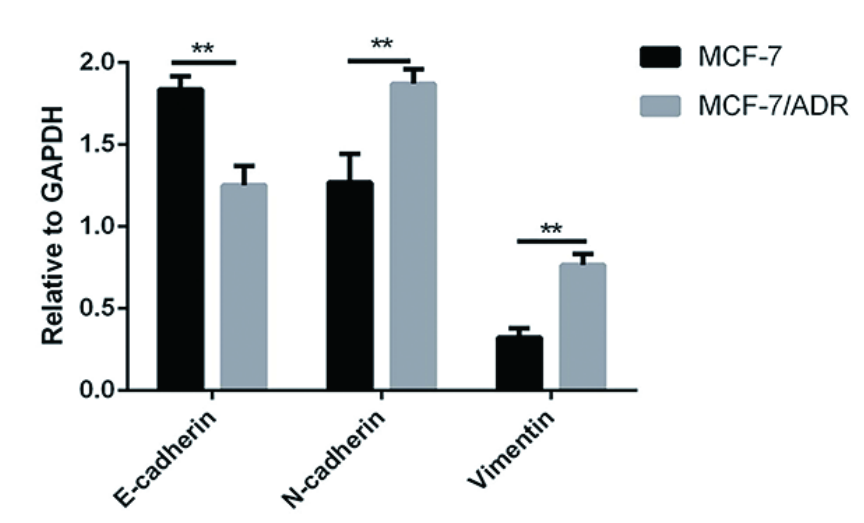

(E)

MCF-7/ADR

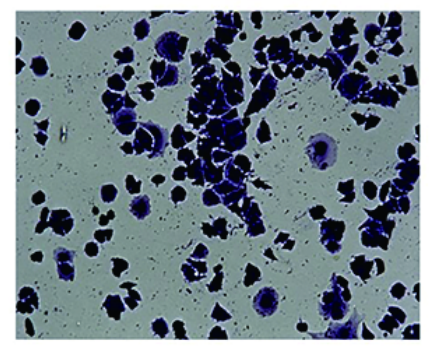

MCF-7/ADR

MCF-7 
(A)

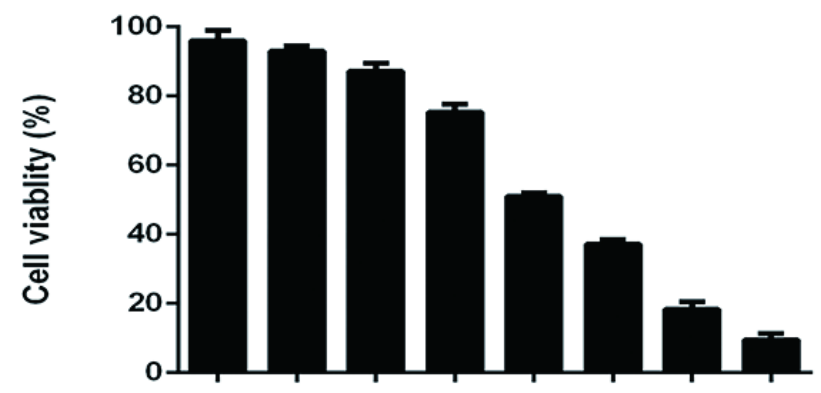

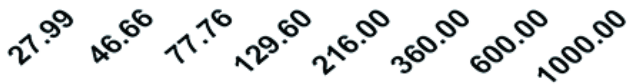

Galunisertib concentration $(\mu \mathrm{M})$

(C)

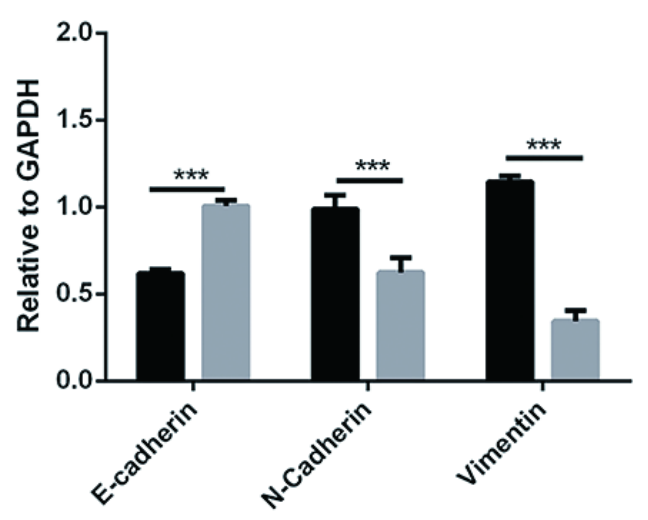

(E)

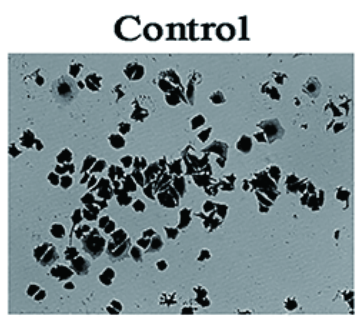

Galunisertib
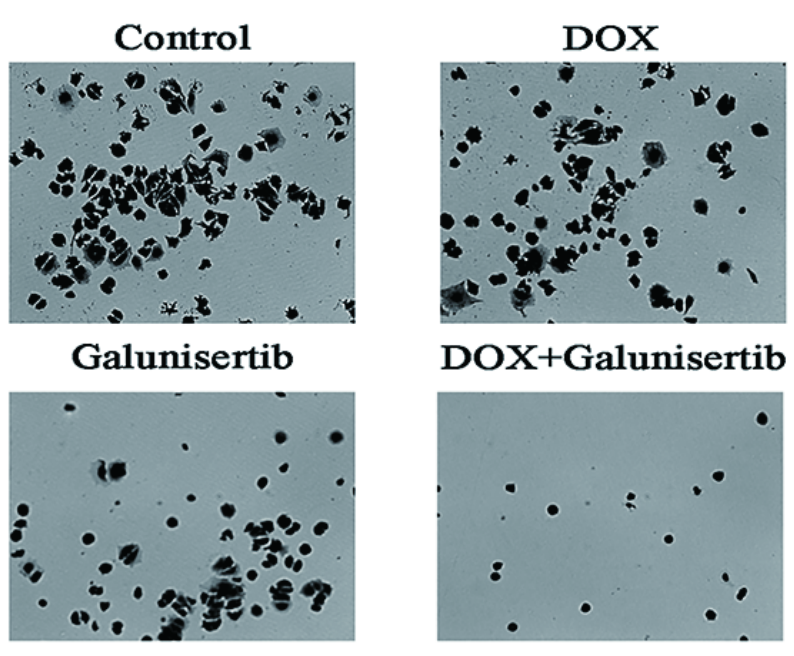

(B)

\section{Control Galunisertib}

E-cadherin

\section{N-cadherin}

Vimentin

GAPDH
(D)

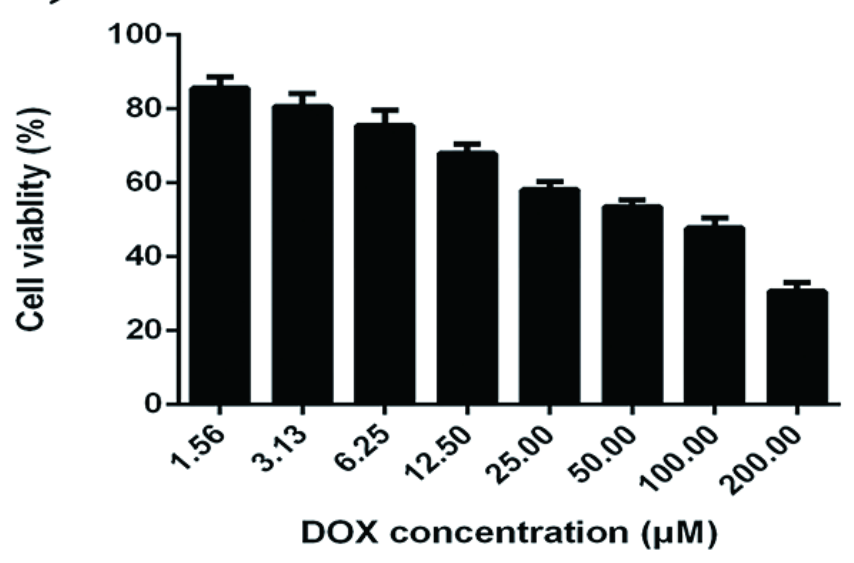

(F)

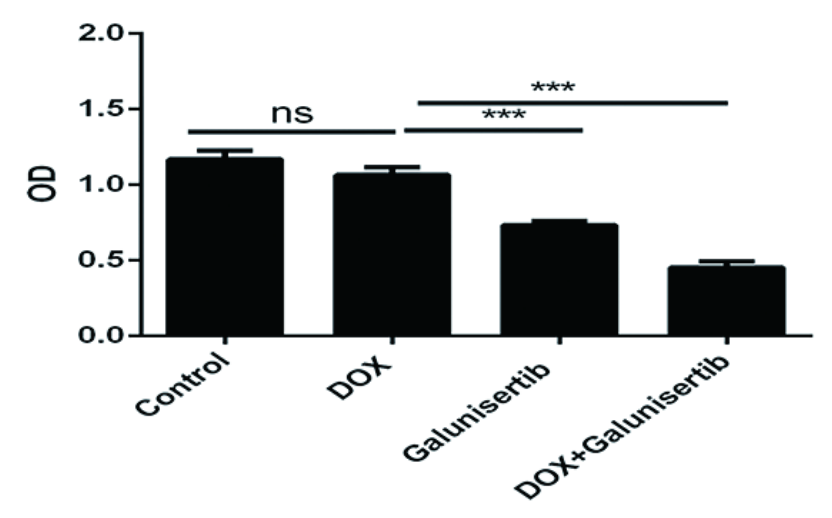

Figure 2

The effect of Galunisertib combined with DOX on cell proliferation and invasion. Cell viability of MCF7/ADR cells after treatment with different Galunisertib concentration (27.9-1000 $\mu \mathrm{M})$. (B) and (C) The effect of $30 \mu \mathrm{M}$ Galunisertib on the expression of EMT marker protein. $N=3,{ }^{* \star} p<0.01,{ }^{* \star *} \mathrm{p}<0.001$ compared with Control group. (D) Cell viability on MCF-7/ADR cells after treatment with $30 \mu \mathrm{M}$ Galunisertib combined with DOX of different concentrations $(1.56-200 \mu \mathrm{M})$. (E) and $(F)$ The invasion 
activity of MCF-7/ADR cells after incubation with DOX (10 $\mu \mathrm{M})$, Galunisertib (30 $\mu \mathrm{M})$, DOX (10 $\mu \mathrm{M})+$ Galunisertib $(30 \mu \mathrm{M})$ over 48 h. $N=3,{ }^{*} \mathrm{p}<0.01,{ }^{* \star} \mathrm{p}<0.001$ compared with DOX group.

(A)

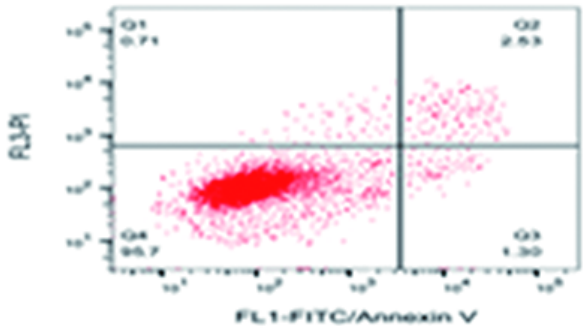

Gatunisertib

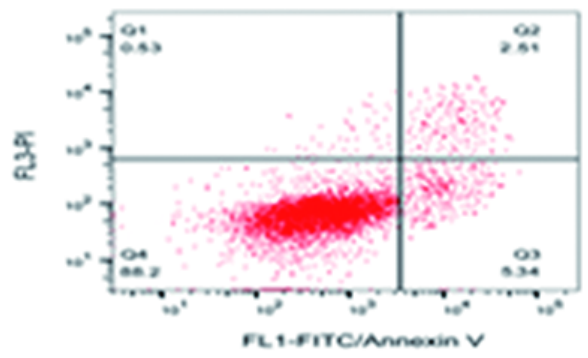

(C)

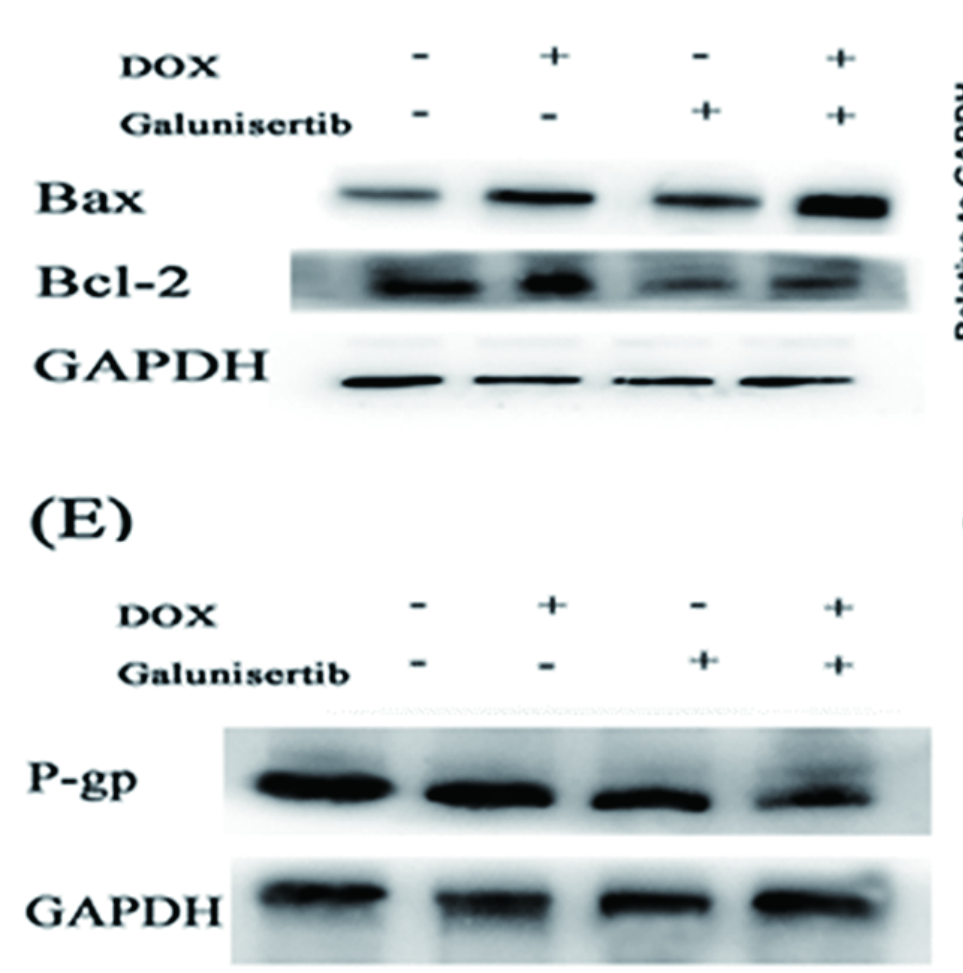

DOX

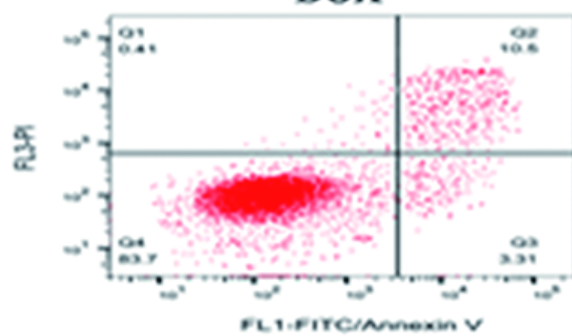

DOX+Galunisertib

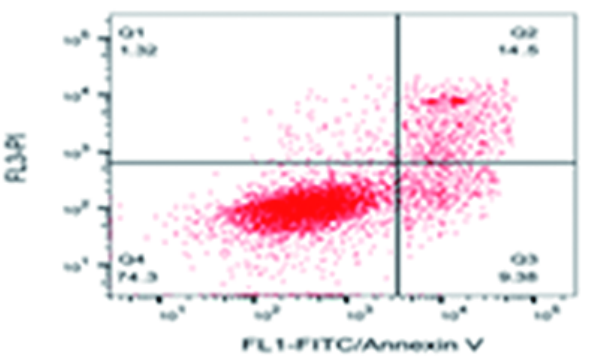

(D)
(B)
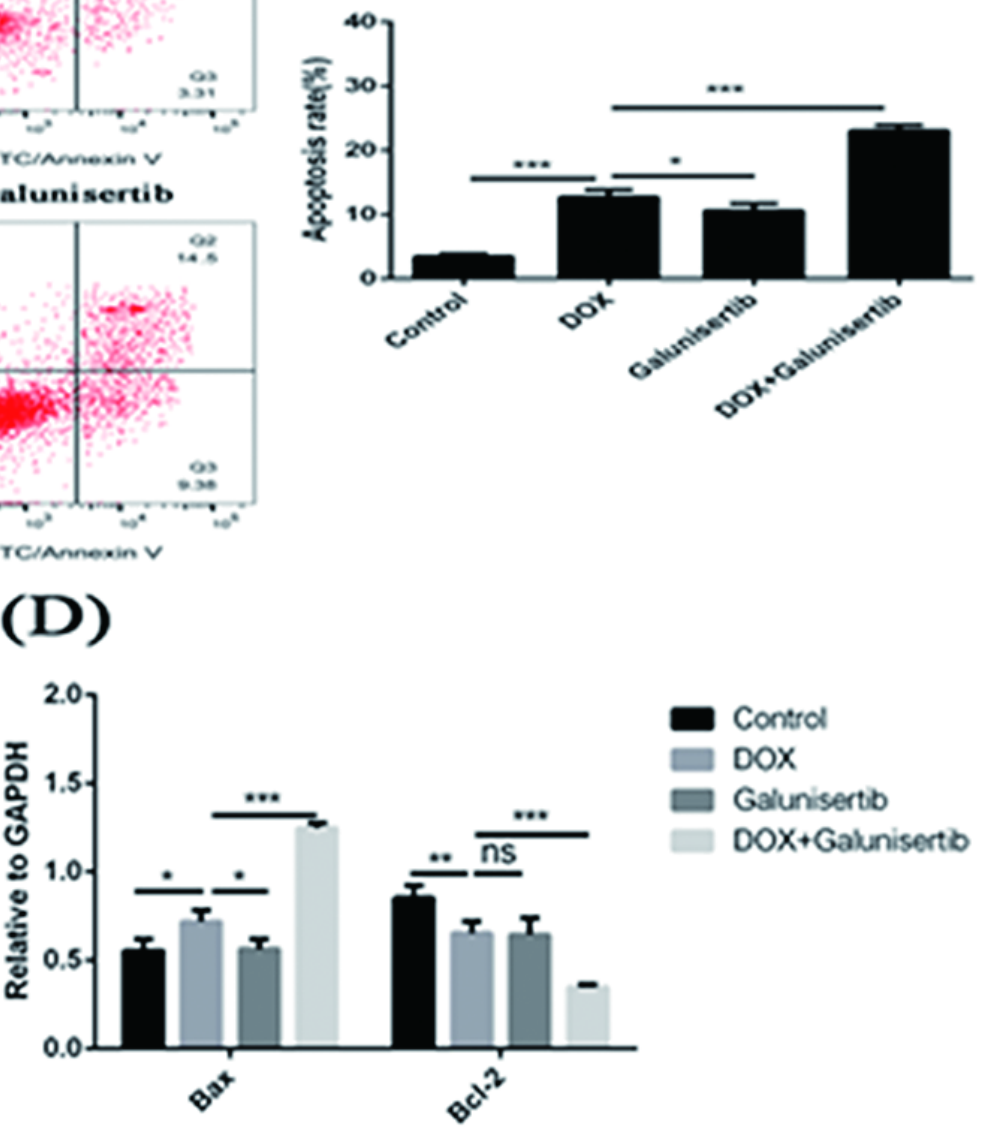

(F)

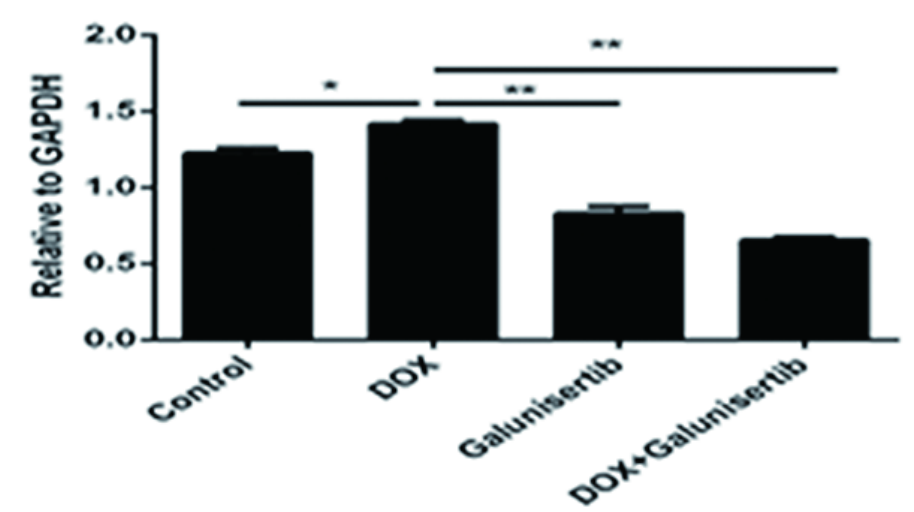

Figure 3

Effect of Galunisertib combined with DOX on apoptosis and expression of drug resistance protein. (A) and (B) The apoptotic rate of MCF-7/ADR cells treated with different groups (DOX, Galunisertib, DOX+ Galunisertib) was measured by flow cytometry. (C) and (D) The expression of Bax and Bcl-2 in MCF- 
7/ADR cells treated with DOX, Galunisertib, DOX+Galunisertib. Relative levels of the proteins were quantified by densitometric analysis. (E) and (F) The expression of P-gp and MRP1 in MCF-7/ADR cells treated with different groups (DOX, Galunisertib, DOX+Galunisertib) . N=3, $* * p<0.01,{ }^{\star \star *} p<0.001$ compared with DOX group.

\section{(A) \\ (B)}
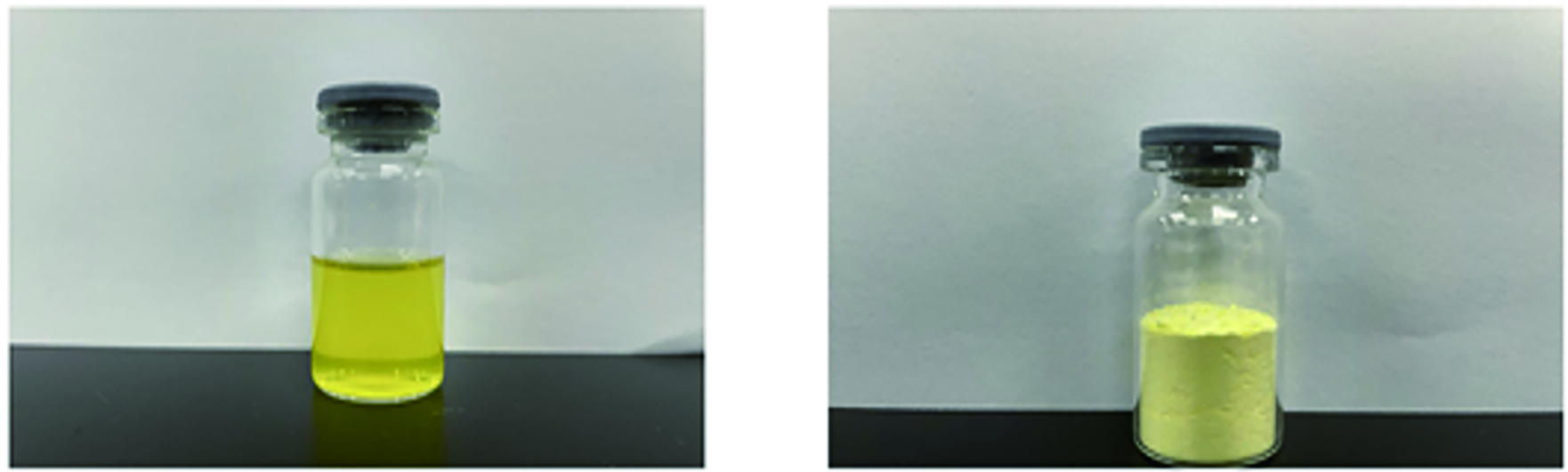

(C)

Stoo Oistribution by Intensty

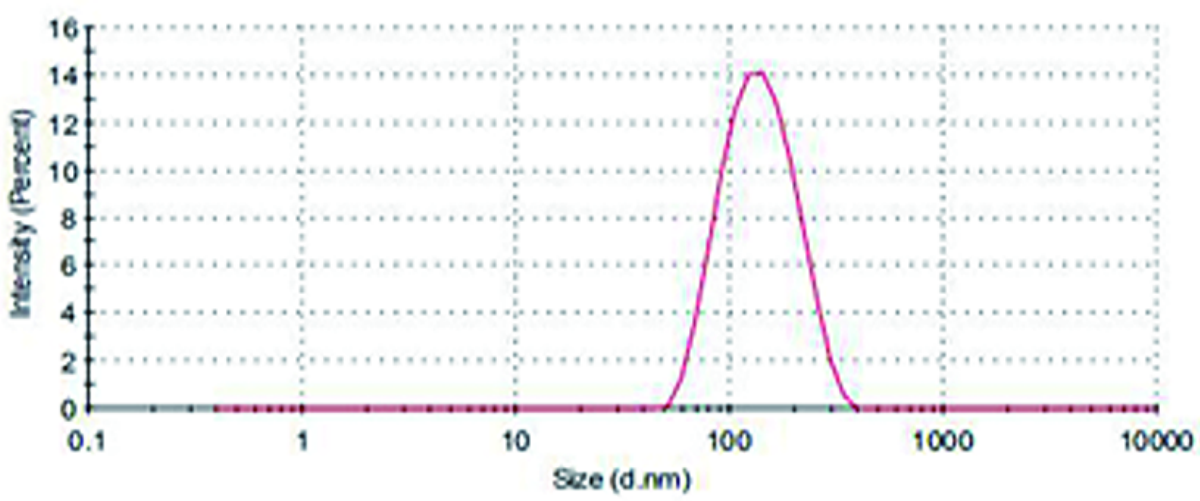

(D)

Record 1: 1

Zeta Potential Distribution

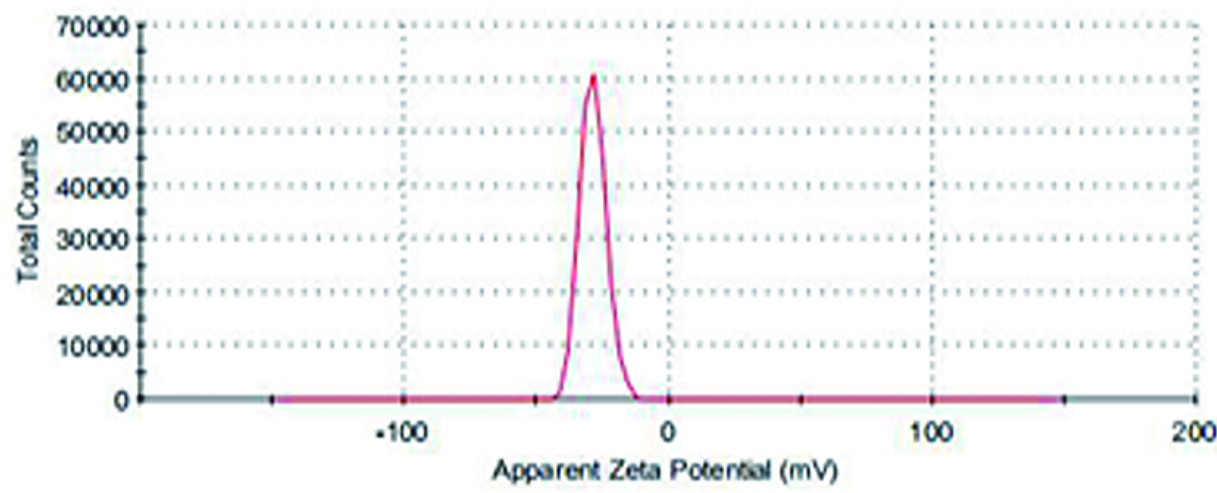

Record 38: 2

Figure 4 
The characterization of E-PLNs. (A) The morphology of E-PLNs. (B) Lyophilized powder of E-PLNs. (C) and (D) The particle size and Zeta potential of E-PLNs was measured by the laser particle size analyzer.
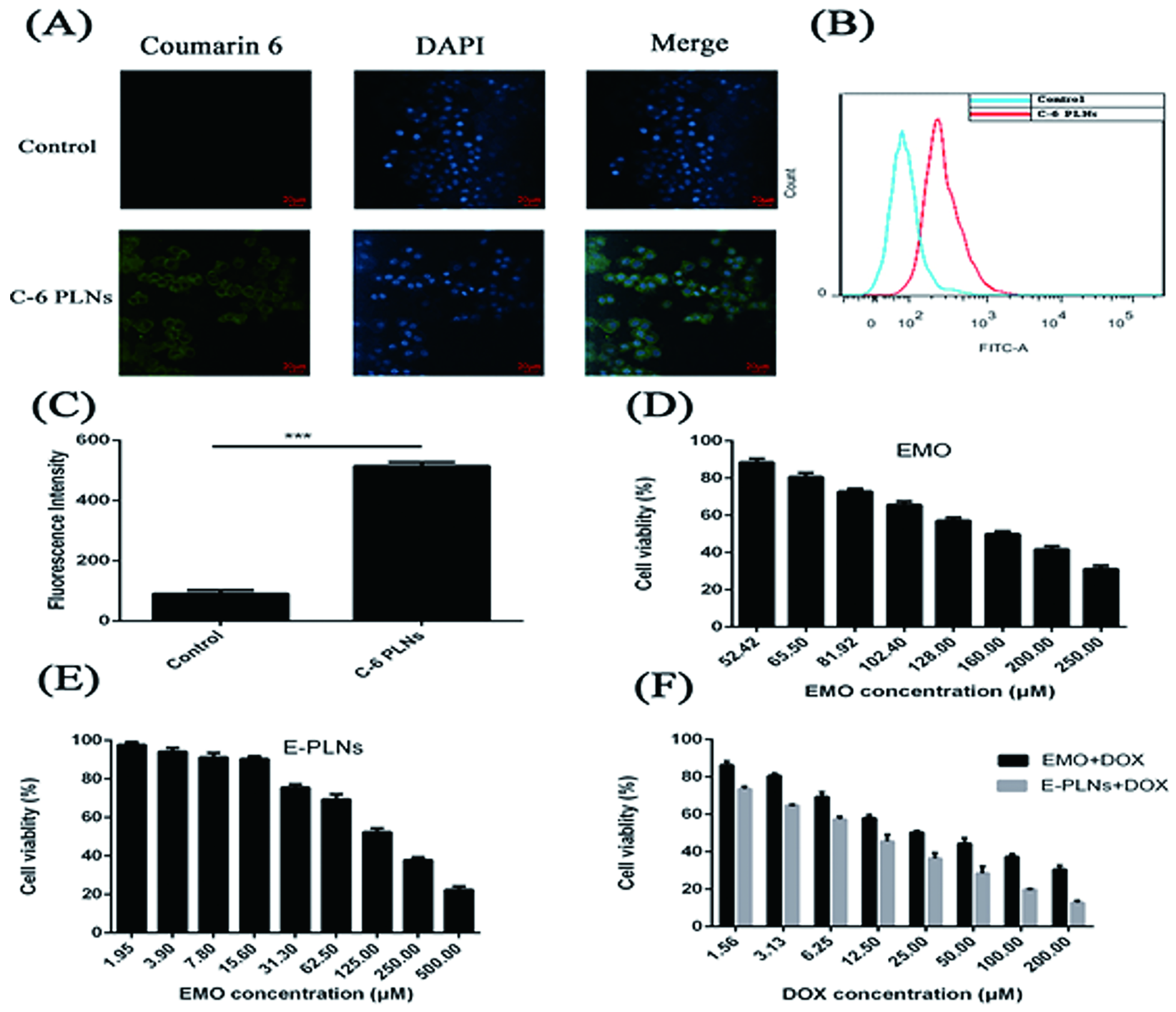

Figure 5

Cellular uptake and cytotoxicity study of E-PLNs. (A) confocal imaging of C-6 PLNs in MCF-7/ADR cells was observed. (B) and (C) Flow cytometry and fluorescence intensity of nanoparticles uptake by MCF7/ADR cells, $N=3,{ }^{* *} p<0.01,{ }^{* *} p<0.001$ compared with Control group. (D) and (E) Cell viability in vitro on MCF-7/ADR cells after treatment with free EMO and E-PLNs. (F) The cell viability of MCF-7/ADR cells treated with $30 \mu \mathrm{M}$ EMO or E-PLNs combined with different concentrations of DOX (1.56-200 $\mu \mathrm{M})$. 


\section{Control}

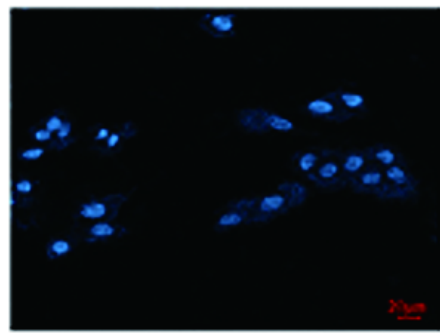

(B)

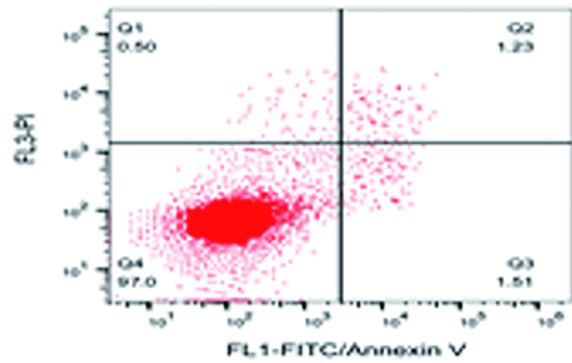

$\mathrm{DOX}+\mathrm{EMO}$

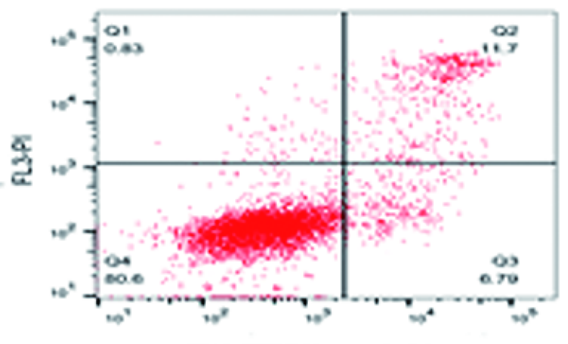

(D)

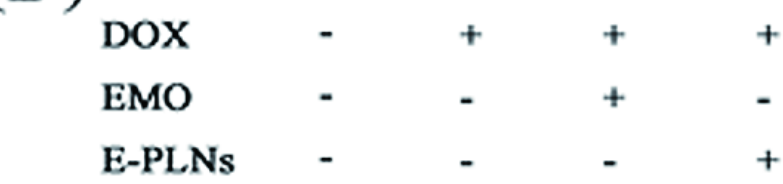

Bax

Bcl-2

GAPDH
DOX

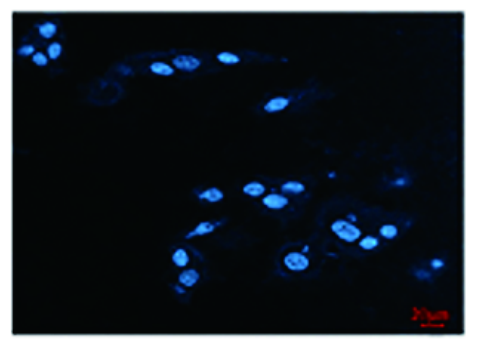

DOX

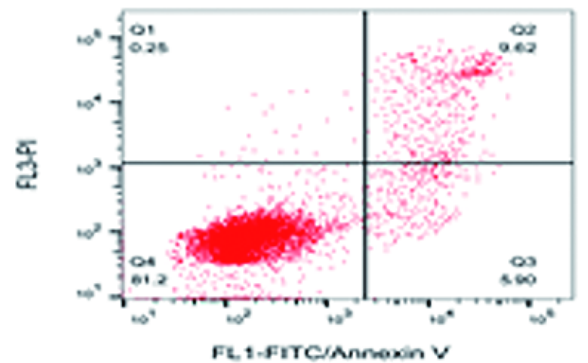

DOX+E-PLNs

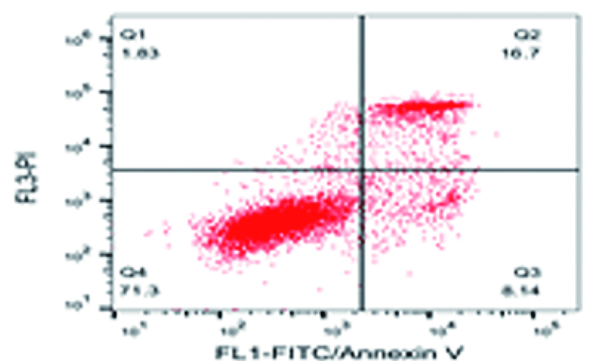

(E)
$\mathrm{DOX}+\mathrm{EMO}$

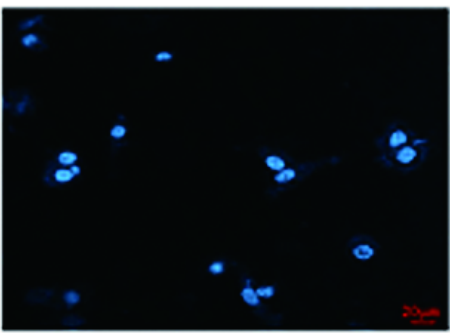

(C)

DOX+E-PLNs

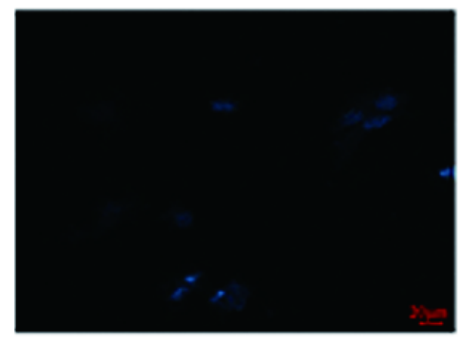

.

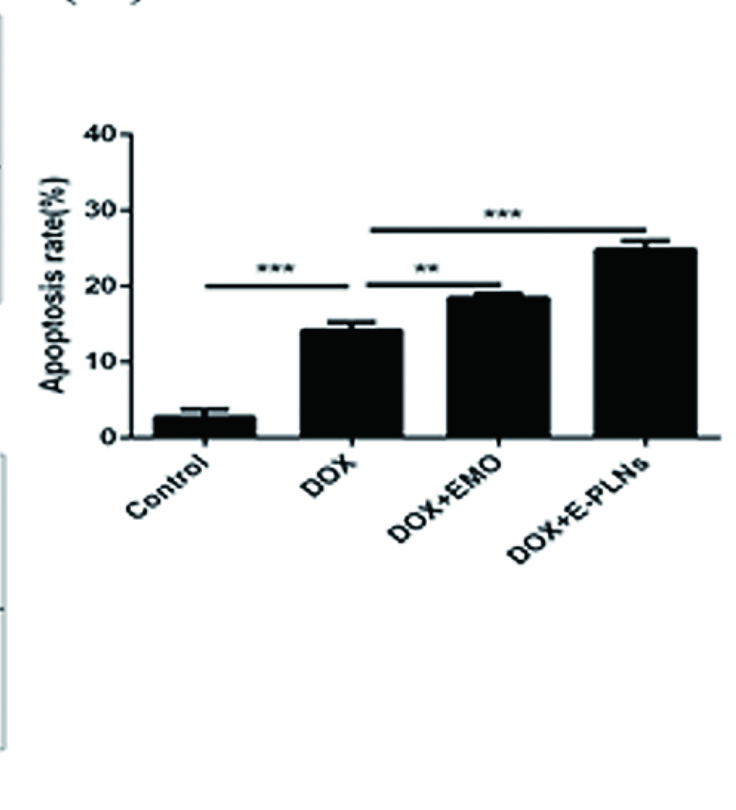

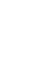

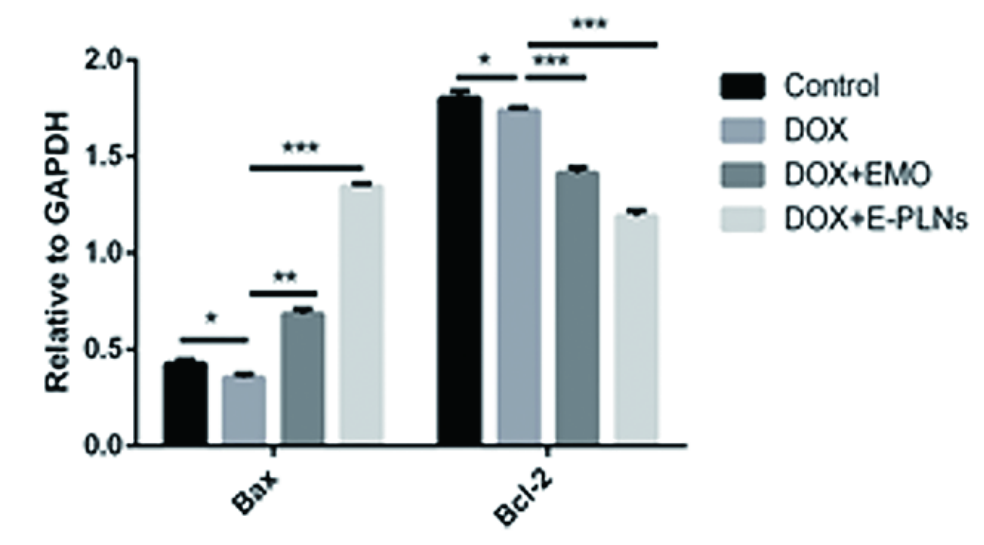

\section{Figure 6}

E-PLNs enhanced DOX induced apoptosis by regulating apoptosis related proteins. (A) Morphological changes of nuclei in MCF-7/ADR cells in the different treated groups using DAPI staining. (B) and (C) Apoptosis assay of MCF-7 cells after incubation with different treated groups. (D) and (E) The expression of Bax and Bcl-2 in MCF-7/ADR cells were determined by Western blot analysis. $N=3,{ }^{\star \star} \mathrm{p}<0.01$, ${ }^{\star \star \star} \mathrm{p}<$ 0.001 compared with DOX group. 
(A)

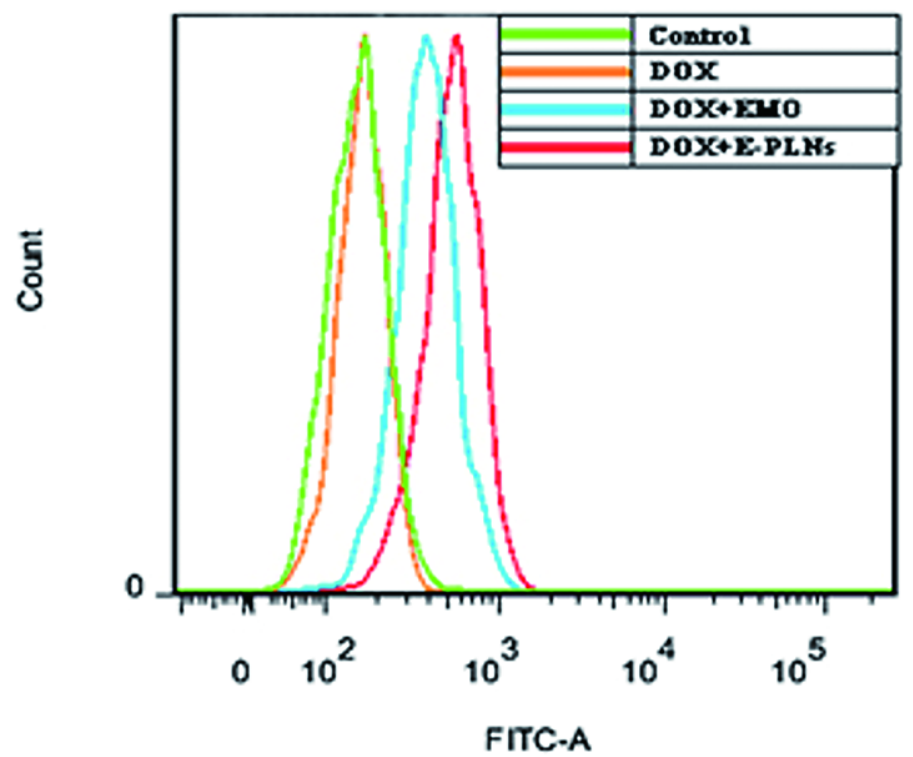

(C)

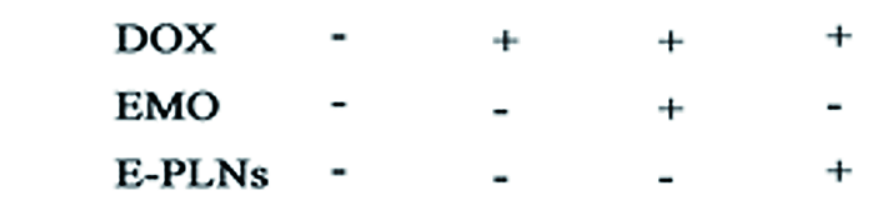

\section{P-gp}

GAPDH
(B)

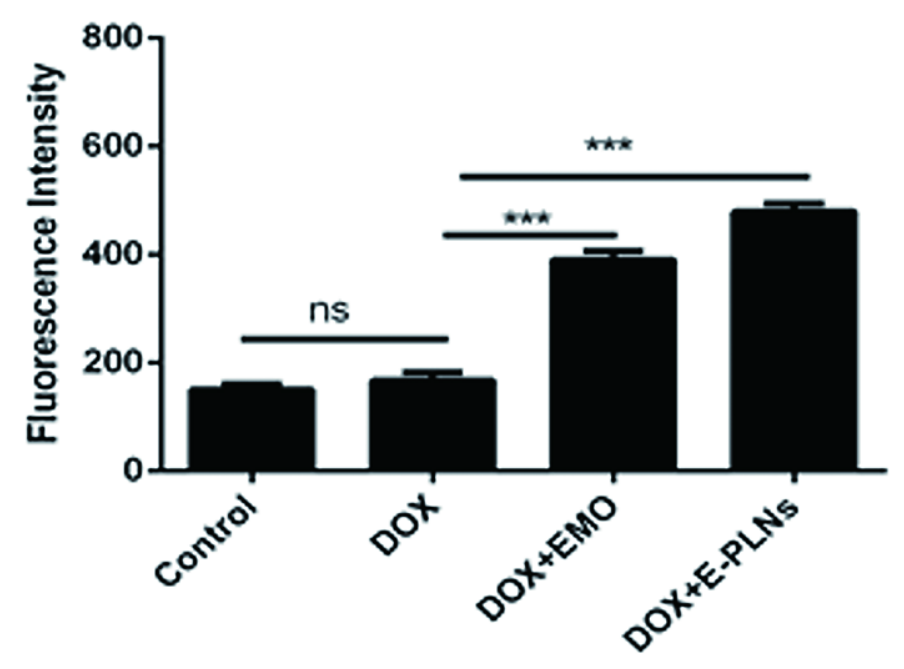

(D)

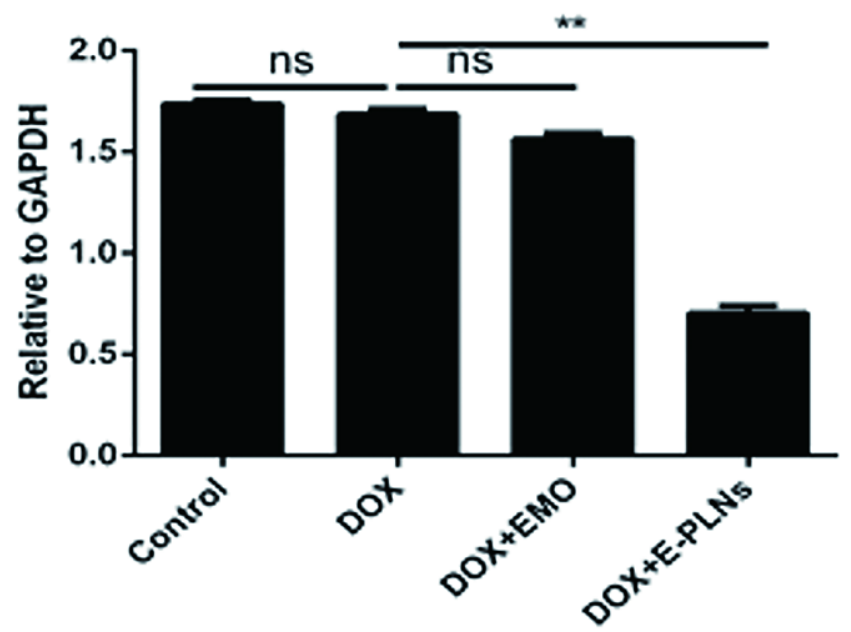

Figure 7

E-PLNs increase the accumulation of DOX by inhibiting the expression of drug resistance protein. $(A)$ and (B) The accumulation of in MCF-7/ADR cells treated with DOX, DOX+EMO, DOX+E-PLNs measured by flow cytometry. (C) and (D) The expression of P-gp in MCF-7/ADR cells detected by Western blotting analysis. $\mathrm{N}=3,{ }^{\star \star} \mathrm{p}<0.01,{ }^{\star \star *} \mathrm{p}<0.001$ compared with DOX group. 
(A)

$\begin{array}{lllll}\text { DOX } & - & + & + & + \\ \text { EMO } & - & - & + & - \\ \text { E-PLNs } & - & - & - & +\end{array}$

E-cadherin

N-cadherin

Vimentin

GAPDE

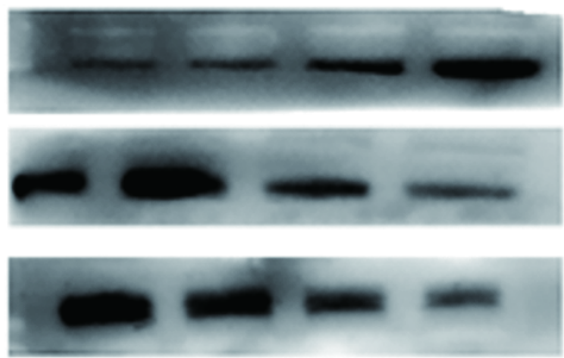

(C)

Control

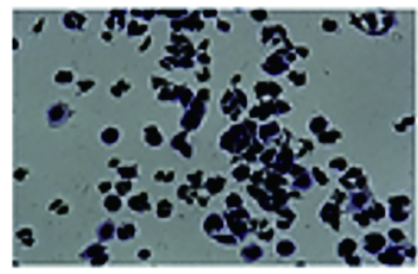

$\mathrm{DOX}+\mathrm{EMO}$

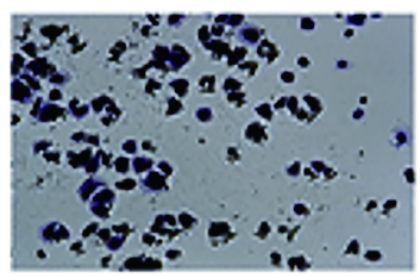

DOX

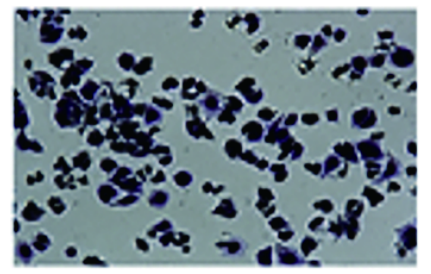

DOX+E-PLNs

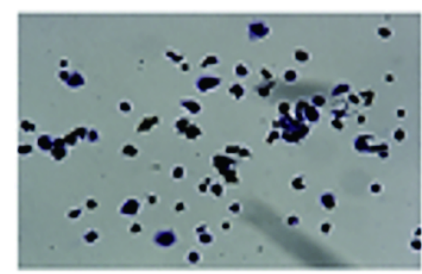

(B)

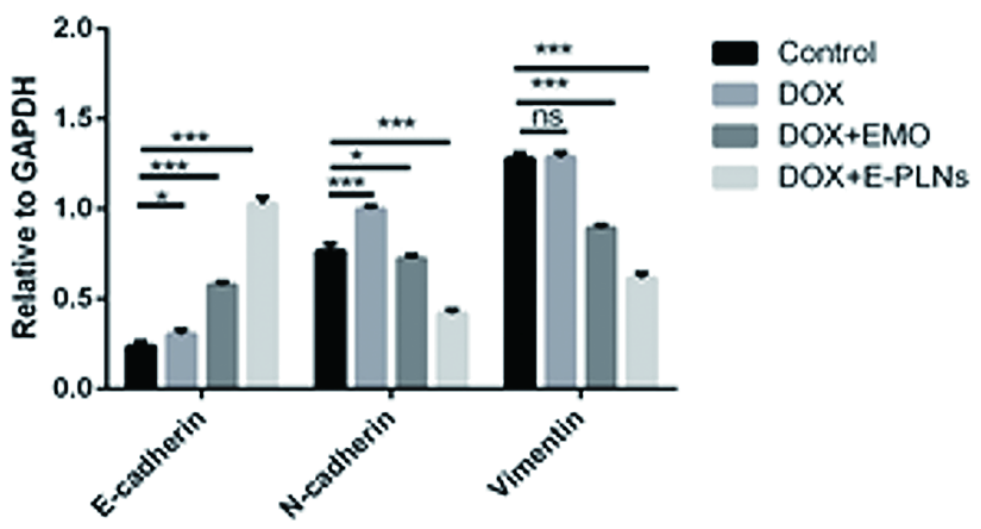

(D)

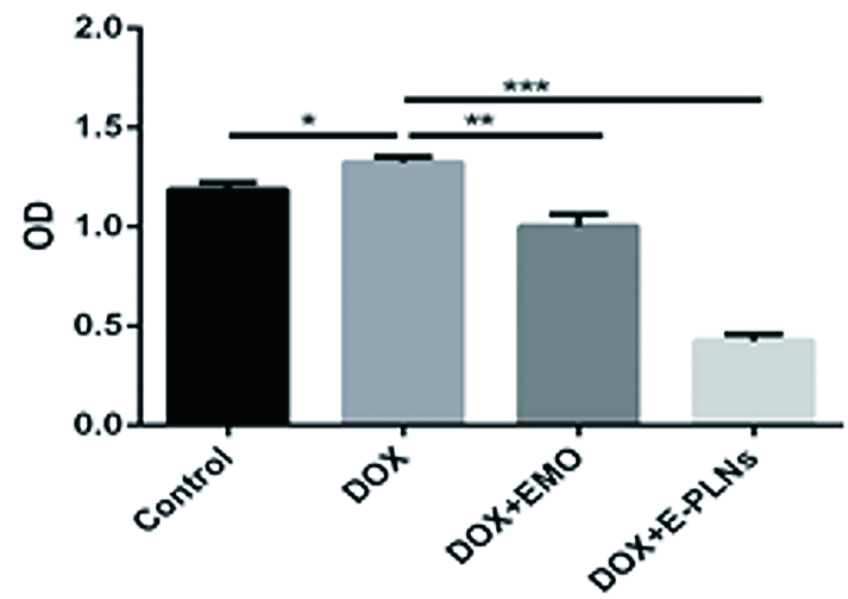

Figure 8

E-PLNs combined with DOX inhibited EMT marker protein expression and cell invasion. (A) and (B) After MCF-7/ADR cells treated with DOX, DOX+EMO, DOX+E-PLNs for $48 \mathrm{~h}$, the expression of E-cadherin, $\mathrm{N}$ cadherin and Vimentin were detected by Western blotting analysis. (C) and (D) The invasion activity of MCF-7/ADR cells after incubation with DOX, DOX+EMO, DOX+E-PLNs for $24 \mathrm{~h}$. N=3, ${ }^{\star \star} \mathrm{p}<0.01$, $* \star \star p<$ 0.001 compared with DOX group. 


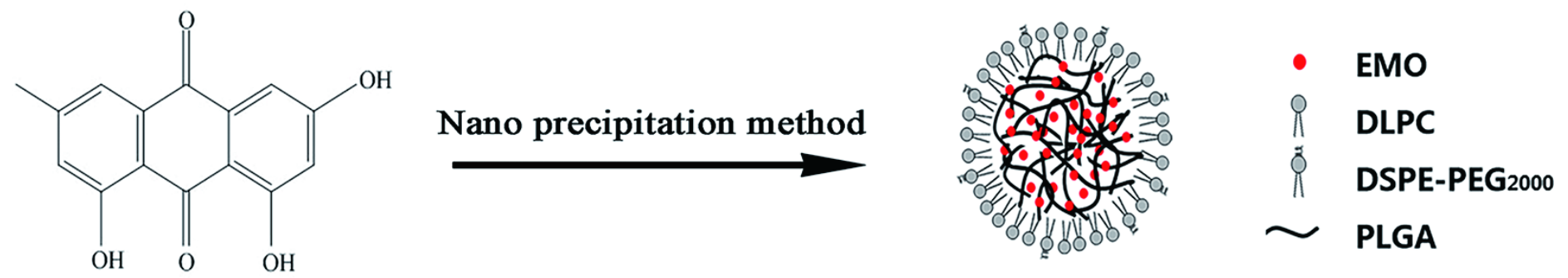

Emodin (EMO)

Emodin-loaded polymer-lipid hybrid nanoparticles (E-PLNs)

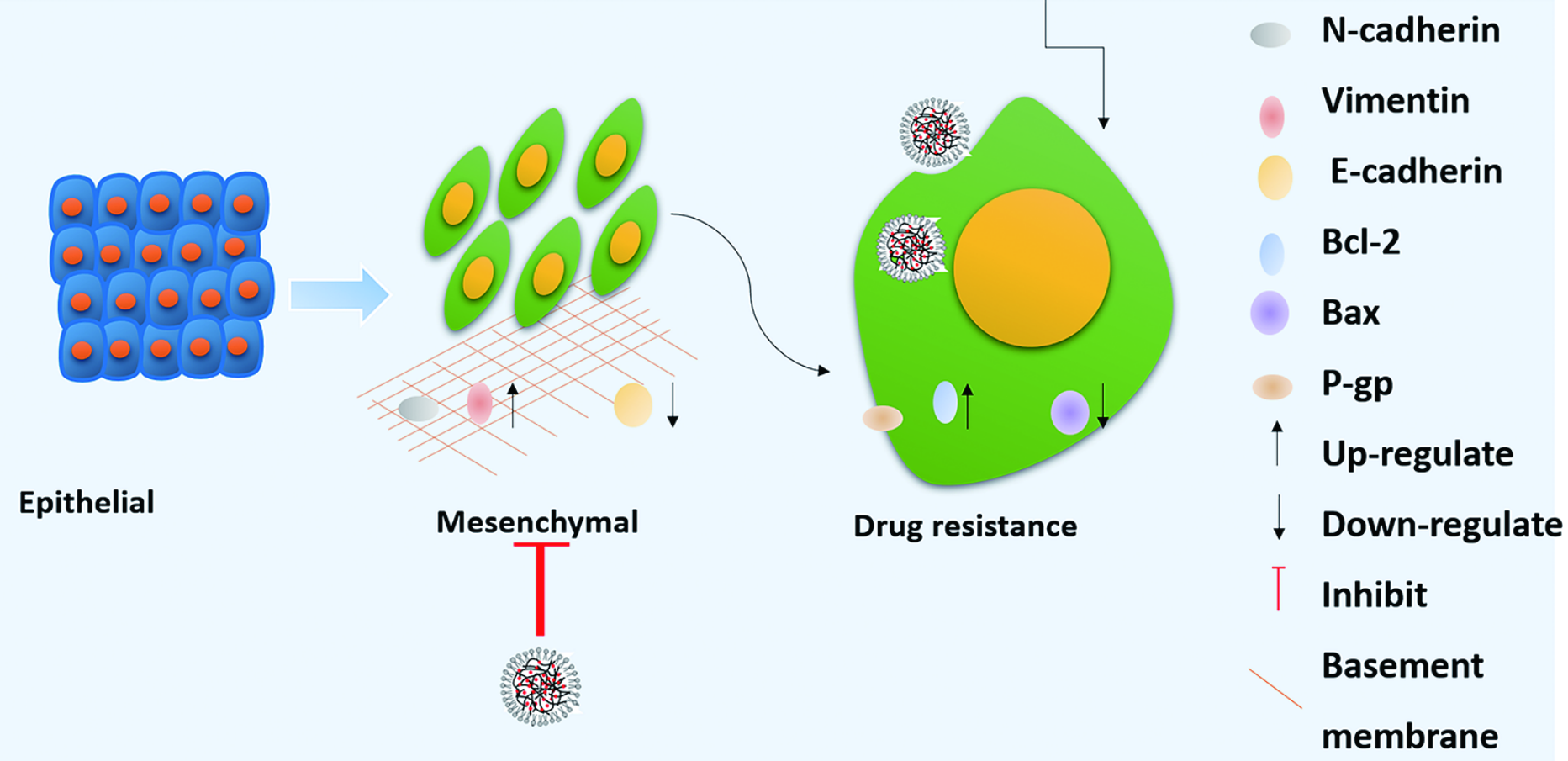

Figure 9

E-PLNs enhance the sensitivity of breast cancer to Doxorubicin by inhibiting epithelial mesenchymal transition. 